This item was submitted to Loughborough's Research Repository by the author.

Items in Figshare are protected by copyright, with all rights reserved, unless otherwise indicated.

\title{
Diffraction by a gap in an infinite permeable breakwater
}

PLEASE CITE THE PUBLISHED VERSION

LICENCE

CC BY-NC-ND 4.0

\section{REPOSITORY RECORD}

Bowen, Matthew K., and P. Mclver. 2019. "Diffraction by a Gap in an Infinite Permeable Breakwater". figshare. https://hdl.handle.net/2134/773. 


\title{
Diffraction by a gap in an infinite permeable breakwater
}

\author{
By M. K. Bowen* and P. McIver ${ }^{\dagger}$
}

\begin{abstract}
The linearized theory of water waves is used to study the diffraction of an incident wave by a gap in a permeable breakwater. Under the assumption that the wavelength is much greater than the thickness, the breakwater is replaced by a thin barrier and a suitable boundary condition applied on the barrier to model the permeability. A new Green's function is used in an application of Green's theorem to obtain an integral equation which is solved numerically to obtain the flow field. An approximate solution valid for large gaps is also given. Results are presented to illustrate the effects of permeability and changes in the angle of wave incidence.
\end{abstract}

\section{Introduction}

This paper is concerned with the diffraction of monochromatic water waves by a permeable breakwater standing in fluid of constant depth. The breakwater is assumed to be straight and to have uniform properties along its length. When the wavelength of the incident wave is much greater than the thickness of the breakwater, then the breakwater may be modelled as a thin vertical barrier which is a geometry more amenable to theoretical investigation. The depth dependence in the flow field is readily factored out so that the problem is reduced to one in two dimensions.

The two-dimensional problem of diffraction of waves by solid (i.e. non-permeable) thin barriers has interpretations in acoustics and electromagnetic waves and has attracted considerable attention in the literature. There is an exact solution for a semi-infinite barrier and this is given in many text books (e.g. Noble, 1988, Chapter 2). For an infinitely-long solid barrier with a gap there is also an exact solution (Carr and Stelzriede, 1952), but this is in terms of Mathieu functions which

\footnotetext{
${ }^{*}$ Undergraduate Student, Department of Mathematical Sciences, Loughborough University, Loughborough, Leics, LE11 3TU, UK

${ }^{\dagger}$ Reader in Applied Mathematics, Department of Mathematical Sciences, Loughborough University, Loughborough, Leics, LE11 3TU, UK
} 
makes computation of the solution rather inconvenient. Consequently, a number of authors (e.g. Gilbert and Brampton (1985), Porter and Chu (1986), Hunt (1990)) have formulated the problem in terms of an integral equation which has then been solved numerically.

Recently attention has turned to the problem of diffraction by a permeable breakwater. Yu (1995) used an approximate procedure to solve the problem of diffraction of a normally-incident wave by a semi-infinite permeable breakwater using a boundary condition based on the formulation of Sollitt and Cross (1972) for time-harmonic motion within a porous medium. Subsequently, McIver (1999) showed that the problem considered by Yu (1995) has an exact solution for all angles of wave incidence. More recently, Lynett et al. (2000) have used numerical and experimental methods to examine the interaction of a solitary wave with a porous breakwater.

Yu and Togashi (1996) considered the diffraction of waves by a permeable breakwater with a gap. Unfortunately, their solution is in error as they assumed that the asymptotic form of the solution at large distances along the barrier applied along the whole length of the barrier. In the present work, this breakwater-gap problem is formulated in terms of an integral equation obtained by an application of Green's theorem that involves a new Green's function for permeable barriers. The integral equation is solved numerically and the solution is used to obtain the diffraction coefficient which describes the far-field behaviour of the scattered waves. The efficiency of the solution is improved by the use of a so-called "embedding" formula which gives the solution for any arbitrary angle of incidence in terms of the solution for one angle of incidence. Further, an approximate solution valid when the gap is much longer than the incident wavelength is presented and compared with the full solution.

\section{Formulation}

Cartesian coordinates $x, y$ and $z$ are chosen with origin in the mean free surface so that the $x$ - and $y$-axes lie in a horizontal plane and the $z$ axis is directed vertically upwards. The breakwater is of thickness $b$ and stands in water of constant depth $h$. It is aligned with the $x$ axis and has a gap of width $2 a$ so that the breakwater occupies the region $|x|>a,|y|<b,-h<z<0$. Horizontal polar coordinates $(r, \theta)$, defined in the standard way, will also be used (see figure 1 ). The fluid motion is assumed to be time harmonic with angular frequency $\omega$ and to be of sufficiently small amplitude for linear theory to be applicable. Exterior to the breakwater the water is assumed to be inviscid and incompressible and the motion to be incompressible and hence it may be described 


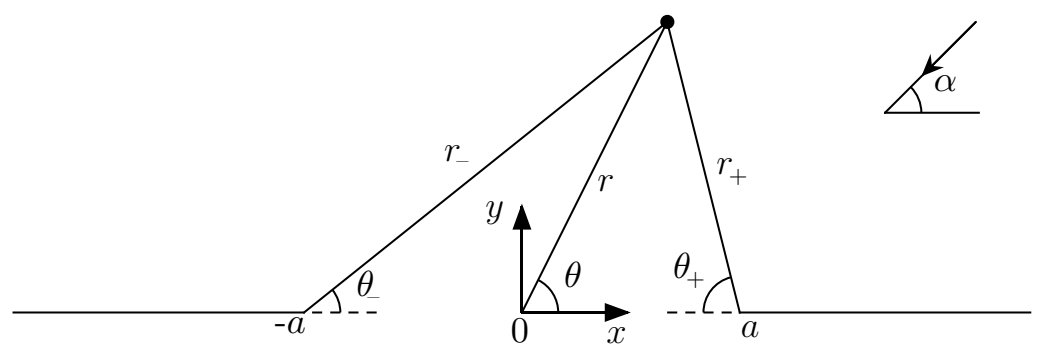

Figure 1: Coordinate systems for the breakwater-gap problem.

by a velocity potential.

An incident wave with wave number $k$ propagates in $y>0$ at an angle $\alpha$ to the $x$ axis, where $k$ is related to the frequency $\omega$ through the dispersion relation

$$
\omega^{2} / g=k \tanh k h
$$

where $g$ is the acceleration due to gravity. The length of the incident wave is assumed to be much greater than the breakwater thickness, so that $k b \ll 1$, and then the thickness may be neglected and the breakwater modelled as a thin permeable barrier. The model of such a breakwater used here is based on the work of Sollitt and Cross (1972) and is described in detail by $\mathrm{Yu}$ (1995); it is also used in related work by McIver (1999). The velocity potential for the flow is written as

$$
\Phi(x, y, z, t)=\operatorname{Re}\left\{\phi_{\mathrm{T}}(x, y) \cosh k(z+h) \mathrm{e}^{-\mathrm{i} \omega t}\right\}
$$

where the complex-valued function $\phi_{\mathrm{T}}(x, y)$ satisfies the Helmholtz equation

$$
\frac{\partial^{2} \phi_{\mathrm{T}}}{\partial x^{2}}+\frac{\partial^{2} \phi_{\mathrm{T}}}{\partial y^{2}}+k^{2} \phi_{\mathrm{T}}=0
$$

in the fluid domain exterior to the breakwater, while the boundary conditions to be satisfied on the breakwater are

$$
\frac{\partial \phi_{\mathrm{T}}}{\partial y}\left(x, 0^{+}\right)=\frac{\partial \phi_{\mathrm{T}}}{\partial y}\left(x, 0^{-}\right)=\beta\left[\phi_{\mathrm{T}}\left(x, 0^{+}\right)-\phi_{\mathrm{T}}\left(x, 0^{-}\right)\right], \quad y=0, \quad|x|>a .
$$

Here the superscript "+" indicates a limit taken from $y>0$ and the superscript "-" indicates a limit taken from $y<0$. The parameter

$$
\beta=\frac{-\mathrm{i} \epsilon}{b(f-\mathrm{i} s)}
$$


where $\epsilon$ is the porosity of the breakwater, $f$ is a non-dimensional friction coefficient, and $s$ is a non-dimensional inertia coefficient.

The total potential $\phi_{\mathrm{T}}$ is decomposed as

$$
\phi_{\mathrm{T}}(x, y)=\mathrm{e}^{-\mathrm{i} k r \cos (\theta-\alpha)}+R_{\alpha} \mathrm{e}^{-i k r \cos (\theta+\alpha)}+\phi^{+}(x, y), \quad y>0,
$$

and

$$
\phi_{\mathrm{T}}(x, y)=T_{\alpha} \mathrm{e}^{-\mathrm{i} k r \cos (\theta-\alpha)}+\phi^{-}(x, y), \quad y<0,
$$

where

$$
R_{\alpha}=\frac{-\mathrm{i} k \sin \alpha}{2 \beta-\mathrm{i} k \sin \alpha} \quad \text { and } \quad T_{\alpha}=1-R_{\alpha}=\frac{2 \beta}{2 \beta-\mathrm{i} k \sin \alpha} .
$$

The first term on the right-hand side of (6) is the incident wave, the second term is the field due to the reflection of the incident wave from an infinitely-long permeable barrier, and the final term is the correction to the field in $y>0$ due to diffraction by the gap. The first term on the righthand side of (7) is the field due to the transmission of the incident wave through an infinitely-long permeable barrier, and the second term is the correction to the field in $y<0$ due to diffraction by the gap. The reflection and transmission coefficients for an infinite barrier, $R_{\alpha}$ and $T_{\alpha}$ respectively, are given by $\mathrm{Yu}$ and Togashi (1996) and also arise in the solution for a semi-infinite barrier obtained by McIver (1999). The diffracted fields $\phi^{+}$and $\phi^{-}$satisfy the radiation conditions

$$
\lim _{r \rightarrow \infty} r^{\frac{1}{2}}\left(\frac{\partial \phi^{ \pm}}{\partial r}-\mathrm{i} k \phi^{ \pm}\right)=0
$$

Continuity of potential in the gap requires that

$$
2 R_{\alpha} \mathrm{e}^{-\mathrm{i} k x \cos \alpha}+\phi^{+}(x, 0)=\phi^{-}(x, 0), \quad|x|<a,
$$

and continuity of velocity in the gap requires

$$
\frac{\partial \phi^{+}}{\partial y}(x, 0)=\frac{\partial \phi^{-}}{\partial y}(x, 0) \equiv U_{\alpha}(x), \quad|x|<a .
$$

The breakwater boundary conditions (4) reduce to

$$
\frac{\partial \phi^{+}}{\partial y}(x, 0)=\frac{\partial \phi^{-}}{\partial y}(x, 0)=\beta\left\{\phi^{+}(x, 0)-\phi^{-}(x, 0)\right\}, \quad|x|>a .
$$

\section{Derivation of an integral equation}

In Appendix I a Green's function $\phi_{0}(x, y ; \xi, \eta)$ is derived that is logarithmically singular at the source point $(\xi, \eta)$, satisfies the Helmholtz equation, the barrier conditions (4) on $y=0$ for all $x$, 
and a radiation condition that specifies outgoing waves. With the source point at $(\xi, \eta), \eta>0$, an application of Greens theorem to $\phi^{+}$and $\phi_{0}$ around a contour consisting of the $y$ axis and an enclosing large semi-circle in $y>0$ gives

$$
\phi^{+}(\xi, \eta)=\frac{\mathrm{i}}{4} \int_{-\infty}^{\infty}\left[\phi^{+}(x, 0) \frac{\partial \phi_{0}}{\partial y}\left(x, 0^{+} ; \xi, \eta\right)-\phi_{0}\left(x, 0^{+} ; \xi, \eta\right) \frac{\partial \phi^{+}}{\partial y}(x, 0)\right] \mathrm{d} x,
$$

and an application of Greens theorem to $\phi^{-}$and $\phi_{0}$ with the contour consisting of the $y$ axis and an enclosing large semi-circle in $y<0$ gives

$$
0=-\frac{\mathrm{i}}{4} \int_{-\infty}^{\infty}\left[\phi^{-}(x, 0) \frac{\partial \phi_{0}}{\partial y}\left(x, 0^{-} ; \xi, \eta\right)-\phi_{0}\left(x, 0^{-} ; \xi, \eta\right) \frac{\partial \phi^{-}}{\partial y}(x, 0)\right] \mathrm{d} x .
$$

The Green's function has been constructed to satisfy the same radiation condition as the diffraction potential, and so the corresponding contributions to the integrals in Green's theorem do not appear in (13)-(14). Addition of (13) and (14) and the use of the continuity of velocity conditions in equations (11), (12) and (45) yield

$$
\begin{aligned}
\phi^{+}(\xi, \eta)=\frac{\mathrm{i}}{4} \int_{-\infty}^{\infty}\left[\left(\phi^{+}(x, 0)-\phi^{-}(x, 0)\right)\right. & \frac{\partial \phi_{0}}{\partial y}(x, 0 ; \xi, \eta) \\
& \left.-\left(\phi_{0}\left(x, 0^{+} ; \xi, \eta\right)-\phi_{0}\left(x, 0^{-} ; \xi, \eta\right)\right) \frac{\partial \phi^{+}}{\partial y}(x, 0)\right] \mathrm{d} x .
\end{aligned}
$$

Finally, equations (12) and (46) are used to eliminate the integrals over the barrier in $|x|>a$, and equations (10) and (11) are used to rewrite the terms involving the diffraction potential, so that

$$
\phi^{+}(\xi, \eta)=-\frac{\mathrm{i}}{4 \beta} \int_{-a}^{a} \frac{\partial \phi_{0}}{\partial y}(x, 0 ; \xi, \eta)\left[2 \beta R_{\alpha} \mathrm{e}^{-\mathrm{i} k x \cos \alpha}+U_{\alpha}(x)\right] \mathrm{d} x, \quad \eta>0,
$$

which is an integral representation for the diffracted field in $y>0$ in terms of the corresponding velocity in the breakwater gap. Similar applications of Green's theorem with the source point at $(\xi,-\eta), \eta>0$, yield with the aid of (61) that

$$
\phi^{-}(\xi,-\eta)=\frac{\mathrm{i}}{4 \beta} \int_{-a}^{a} \frac{\partial \phi_{0}}{\partial y}(x, 0 ; \xi, \eta)\left[2 \beta R_{\alpha} \mathrm{e}^{-\mathrm{i} k x \cos \alpha}+U_{\alpha}(x)\right] \mathrm{d} x, \quad \eta>0,
$$

and therefore

$$
\phi^{-}(\xi,-\eta)=-\phi^{+}(\xi, \eta) .
$$

Substitution of the above expressions for $\phi^{ \pm}$into the potential continuity condition (10) gives the integral equation

$$
\int_{-a}^{a} h(|x-t|) V_{\alpha}(t) \mathrm{d} t=f_{\alpha}(x)
$$


for $V_{\alpha}$, where

$$
\begin{gathered}
h(|x-t|)=\frac{\mathrm{i}}{4 \beta} \frac{\partial \phi_{0}}{\partial y}(t, 0 ; x, 0), \\
V_{\alpha}(t)=2 \beta \mathrm{e}^{-\mathrm{i} k t \cos \alpha}+\frac{U_{\alpha}(t)}{R_{\alpha}}
\end{gathered}
$$

and

$$
f_{\alpha}(x)=\mathrm{e}^{-\mathrm{i} k x \cos \alpha}
$$

An integral representation for the kernel $h$ follows from equation (59).

In the solid barrier limit $\beta \rightarrow 0$ then

$$
R_{\alpha} \rightarrow 1, \quad V_{\alpha} \rightarrow U_{\alpha}
$$

and from equations (59) and (50)

$$
h(|x-t|) \rightarrow \frac{\mathrm{i}}{2} H_{0}^{(1)}(k|x-t|),
$$

where $H_{0}^{(1)}$ denotes a Hankel function of the first kind, so that the integral equation (19) reduces to the equation for the solid barrier given, for example, by Porter and Chu (1986, equation 2.1).

\section{The diffraction coefficient}

Of particular interest is the behaviour of the diffracted field at large distances from the gap. Application of an asymptotic result given by Noble (1988, equation 1.71) gives, after a change of notation in (59),

$$
\frac{\partial \phi_{0}}{\partial y}(t, 0 ; x, y) \sim 2 \beta\left(\frac{2 k}{\pi r}\right)^{\frac{1}{2}} \frac{\mathrm{e}^{\mathrm{i}\left(k r-\frac{3 \pi}{4}\right)-\mathrm{i} k t \cos \theta}}{2 \beta-\mathrm{i} k \sin \theta} \sin \theta \quad \text { as } k r \rightarrow \infty
$$

and hence it follows from (16) that

$$
\phi^{+}(x, y) \sim \frac{\mathrm{e}^{\mathrm{i}\left(k r-\frac{3 \pi}{4}\right)}}{(2 \pi k r)^{\frac{1}{2}}} G(\theta, \alpha) \quad \text { as } k r \rightarrow \infty
$$

where

$$
G(\theta, \alpha)=R_{\theta} R_{\alpha} H(\theta, \alpha), \quad 0<\theta<\pi,
$$

and

$$
H(\theta, \alpha)=\int_{-a}^{a} V_{\alpha}(t) f_{\theta}(t) \mathrm{d} t .
$$

Thus, once the integral equation (19) has been solved for $V_{\alpha}$ the so-called "diffraction coefficient" $G(\theta, \alpha)$ follows from (27). The diffraction coefficient describes the angular variation of the diffracted 
waves in the far field. Note that the above calculation is for the waves in the upper-half plane; the field for the lower-half plane follows from (18).

From its definition and the properties of the integral equation (19)

$$
\begin{aligned}
H(\theta, \alpha) & =\int_{-a}^{a} V_{\alpha}(t) f_{\theta}(t) \mathrm{d} t \\
& =\int_{-a}^{a} V_{\alpha}(t)\left\{\int_{-a}^{a} h(|x-t|) V_{\theta}(x) \mathrm{d} x\right\} \mathrm{d} t \\
& =\int_{-a}^{a} V_{\theta}(x)\left\{\int_{-a}^{a} h(|x-t|) V_{\alpha}(t) \mathrm{d} t\right\} \mathrm{d} x \\
& =\int_{-a}^{a} V_{\theta}(x) f_{\alpha}(x) \mathrm{d} x=H(\alpha, \theta)
\end{aligned}
$$

from which follows the reciprocity relation

$$
G(\theta, \alpha)=G(\alpha, \theta)
$$

That is, the far-field form of the diffracted waves in the direction $\theta$ due to waves incident at angle $\alpha$ is the same as the far-field form in the direction $\alpha$ due to waves incident at angle $\theta$

The difference-kernel integral equation (19) and the function $H(\theta, \alpha)$ have the same structure as the equivalent objects in the problem of diffraction of waves by a gap in a solid breakwater. One consequence of this is that, for a fixed $k a$, the solution for any angle of wave incidence can be calculated entirely in terms of the solution for one angle of wave incidence (Porter and Chu, 1986). In particular, $H(\theta, \alpha)$ may be expressed in terms of $H(\theta, 0)$ and so the integral equation need only be solved for $\alpha=0$. It follows immediately from such a so-called embedding formula (Porter and Chu, 1986, equations 2.15-2.16) that the diffraction coefficient

$$
G(\theta, \alpha)=R_{\theta} R_{\alpha} \frac{\hat{H}(\alpha) \hat{H}(\theta)-\hat{H}(\pi-\alpha) \hat{H}(\pi-\theta)}{2 H(0,0)(\cos \alpha+\cos \theta)}
$$

where

$$
\hat{H}(\theta)=(1+\cos \theta) H(\theta, 0) .
$$

Equation (31) is undefined for $\theta=\pi-\alpha$, but an application of L'Hôpital's rule yields

$$
G(\pi-\alpha, \alpha)=-R_{\alpha}^{2} \frac{\hat{H}(\alpha) \hat{H}^{\prime}(\pi-\alpha)+\hat{H}(\pi-\alpha) \hat{H}^{\prime}(\alpha)}{2 H(0,0) \sin \alpha} .
$$

One feature of the embedding formulae obtained by Porter and Chu (1986) for diffraction by a gap in a infinite barrier is the role of the problem of diffraction by an insular barrier, that is an isolated barrier of finite length. When the barriers are solid, the insular barrier problem may be 
formulated in terms of an integro-differential equation which is "complementary" to the integral equation for the gap problem, in the sense that the solution of the integral equation for the gap problem with zero angle of incidence is sufficient to obtain the solutions for both the gap problem and the insular barrier problem for any angle of incidence. For the permeable barrier problems this is not the case. There is a complementary integro-differential equation to the integral equation (19) but, as far as the authors are aware, it has no meaningful physical interpretation except in the limit $\beta \rightarrow 0$.

\section{Numerical solution}

The integral equation (19) may be solved numerically as follows. In the integral equation make the changes of variables $x=a \cos u$ and $t=a \cos v$ to get

$$
\frac{\mathrm{i}}{4 \beta} \int_{0}^{\pi} \frac{\partial \phi_{0}}{\partial y}(a \cos v, 0 ; a \cos u, 0) W_{\alpha}(u) \mathrm{d} v=\mathrm{e}^{-\mathrm{i} k a \cos u \cos \alpha},
$$

where

$$
W_{\alpha}(u)=a V_{\alpha}(a \cos u) \sin u
$$

Divide the interval $[0, \pi]$ into $\mathrm{N}$ elements with end-points $\left\{u_{i}=(i-1) \pi / N ; i=1,2, \ldots, N+1\right\}$ and mid-points $\left\{\hat{u}_{i}=\left(i-\frac{1}{2}\right) \pi / N ; i=1,2, \ldots, N\right\}$ and collocate at the mid-points to get

$$
\frac{\mathrm{i}}{4 \beta} \sum_{j=1}^{N} W_{\alpha}\left(\hat{u}_{j}\right) \int_{u_{j}}^{u_{j+1}} \frac{\partial \phi_{0}}{\partial y}\left(a \cos v, 0 ; a \cos \hat{u}_{i}, 0\right) \mathrm{d} v=\mathrm{e}^{-\mathrm{i} k a \cos \hat{u}_{i} \cos \alpha}, \quad i=1,2, \ldots, N .
$$

The integrals appearing in (36) must be evaluated numerically; the evaluation of the integrand is discussed in Appendix II.

Equations (36) may now be readily solved for each $W_{\alpha}\left(\hat{u}_{j}\right)$ and the diffraction coefficient follows directly from (27) and is

$$
G(\theta, \alpha) \approx R_{\theta} R_{\alpha} \sum_{j=1}^{N} W_{\alpha}\left(\hat{u}_{j}\right) \int_{u_{j}}^{u_{j+1}} \mathrm{e}^{-\mathrm{i} k a \cos v \cos \theta} \mathrm{d} v, \quad 0<\theta<\pi .
$$

An alternative is to calculate the $W_{\alpha}\left(\hat{u}_{j}\right)$ for angle of incidence $\alpha=0$ and then use

$$
H(\theta, 0) \approx \sum_{j=1}^{N} W_{0}\left(\hat{u}_{j}\right) \int_{u_{j}}^{u_{j+1}} \mathrm{e}^{-\mathrm{i} k a \cos v \cos \theta} \mathrm{d} v, \quad 0<\theta<\pi
$$

in the embedding formulae (31) and (33) to calculate the diffraction coefficient for any angle of incidence. The wave field at any point in the fluid domain may be computed from (16) and (18). 


\section{Large-gap approximation}

A large-gap (or, equivalently, a high-frequency) approximation to the breakwater gap problem may be derived under the assumption that the ends of the gap are sufficiently far apart, on the scale of the wavelength, to be non-interacting. This approach was adopted by Penney and Price (1952) for the solid breakwater with a gap. With polar coordinates $\left(r_{ \pm}, \theta_{ \pm}\right)$defined as in figure 1 , the approximation to the total potential is (Jones, 1986, §9.25)

$$
\phi_{\mathrm{T}}(r, \theta ; \alpha) \approx \mathrm{e}^{-\mathrm{i} r \cos (\theta-\alpha)}+\mathrm{e}^{\mathrm{i} k a \cos \alpha} \phi\left(r_{-}, \theta_{-} ; \alpha\right)+\mathrm{e}^{-\mathrm{i} k a \cos \alpha} \phi\left(r_{+}, \theta_{+} ; \pi-\alpha\right)
$$

where $\phi(r, \theta ; \alpha)$ is the solution for diffraction of a wave incident at an angle $\alpha$ to the $x$ axis, by a semi-infinite barrier that occupies the negative $x$ axis. An approximation to the diffraction coefficient $G(\theta, \alpha)$, defined in (26), is obtained from examination of the far-field forms of the last two terms in (39) which follow from results given by McIver (1999). As $r / a \rightarrow \infty$ for $0<\theta<\pi$

$$
r_{ \pm} \sim r \mp a \cos \theta, \quad \theta_{-} \sim \theta, \quad \theta_{+} \sim \pi-\theta,
$$

and so, after substitution from McIver (1999, equation 19), it is found that

$$
\begin{aligned}
G(\theta, \alpha) \approx & \frac{\mathrm{i} k \sin \theta \sin \alpha}{(\cos \theta+\cos \alpha)(2 \beta-\mathrm{i} k \sin \theta)}\left\{\mathrm{e}^{\mathrm{i} k a(\cos \theta+\cos \alpha)} \frac{K_{+}(k \cos (\pi-\theta))}{K_{+}(k \cos \alpha)}\right. \\
& \left.-\mathrm{e}^{-\mathrm{i} k a(\cos \theta+\cos \alpha)} \frac{K_{+}(k \cos \theta)}{K_{+}(k \cos (\pi-\alpha))}\right\}, \quad 0<\theta<\pi,
\end{aligned}
$$

where

$$
K_{+}(z)=\left(1+\frac{z}{q}\right)^{1 / 2}\left(\frac{q+2 \beta}{k}\right)^{\frac{\mathrm{i}}{2 \pi} \log \left[\frac{q-z}{q+z}\right]} \exp \left\{\frac{2 \mathrm{i} \beta}{\pi k} \int_{\pi / 2}^{\cos ^{-1}(z / k)} \frac{\theta^{\prime} \cos \theta^{\prime} \mathrm{d} \theta^{\prime}}{\cos ^{2} \theta^{\prime}-(q / k)^{2}}\right\},
$$

the principal branch of the natural logarithm is to be taken and

$$
q^{2}=k^{2}+4 \beta^{2}, \quad 0<\arg q<\pi
$$

Due to the appearance of complex quantities, computer algebra systems like Maple or Mathematica are particularly suitable for computing (41).

\section{Results}

Graphical results are now presented in figures 2 and 3 to illustrate the effects of permeability on the diffraction coefficient defined in equation (27) and the accuracy of the large-gap approximation 
given in equation (41). In the figures results for a solid barrier, that is $\beta=0$, are compared with those for a permeable barrier with $\beta / k=(1-\mathrm{i}) / 4$. This last value of the permeability parameter $\beta$ was one of those used by both Yu (1995) and McIver (1999).

The effect of permeability is to give a general decrease in diffraction effects. It should be born in mind that the permeability also affects the reflection and transmission coefficients appearing in the full solutions of equations (6) and (7); the diffraction coefficient describes the far-field behaviour of only the last terms in these equations. One feature of the results is that as $\theta$ approaches zero or $\pi$ the diffraction coefficient tends to zero for a permeable barrier but the limit is non-zero for a solid barrier. This behaviour is confirmed in the large-gap solution (41).

These, and other computations, suggest that the large-gap approximation performs least well for the solid barrier and the agreement is worse for shallow angle of incidence relative to the barriers. The agreement improves as $k a$ increases which can be interpreted as the gap width increasing for a fixed incident wavelength, or as the incident wavelength decreasing for a fixed gap width.

\section{Acknowledgement}

This work was supported by Nuffield Foundation Undergraduate Research Bursary URB/00034/A.

\section{Appendix I Derivation of the Greens function}

Here logarithmically singular solutions (that is Green's functions) of the Helmholtz equation are derived that satisfy the boundary conditions (12). Thus, it is required to construct solutions $\phi_{0}(x, y ; \xi, \eta)$ of the Helmholtz equation

$$
\frac{\partial^{2} \phi_{0}}{\partial x^{2}}+\frac{\partial^{2} \phi_{0}}{\partial y^{2}}+k^{2} \phi_{0}=0
$$

that are logarithmically singular at $(\xi, \eta)$ and satisfy

$$
\begin{gathered}
\frac{\partial \phi_{0}}{\partial y}\left(x, 0^{+} ; \xi, \eta\right)=\frac{\partial \phi_{0}}{\partial y}\left(x, 0^{-} ; \xi, \eta\right), \text { for all } x, \\
\frac{\partial \phi_{0}}{\partial y}\left(x, 0^{+} ; \xi, \eta\right)=\beta\left\{\phi_{0}\left(x, 0^{+} ; \xi, \eta\right)-\phi_{0}\left(x, 0^{-} ; \xi, \eta\right)\right\}, \text { for all } x,
\end{gathered}
$$

and the radiation condition

$$
\lim _{k r \rightarrow \infty} r^{\frac{1}{2}}\left(\frac{\partial \phi_{0}}{\partial r}-\mathrm{i} k \phi_{0}\right)=0
$$


Here the correct singularity is obtained by requiring

$$
\phi_{0} \sim H_{0}^{(1)}(k R) \quad \text { as } k R \rightarrow 0,
$$

where $H_{0}^{(1)}(k R)$ is the fundamental source solution for the Helmholtz equation in an unbounded medium and

$$
R=\left[(x-\xi)^{2}+\left(y-\eta^{2}\right)\right]^{1 / 2}
$$

is the distance between the source point $(\xi, \eta)$ and the field point $(x, y)$. For future use it is noted that the Helmholtz source has the integral representation (McIver and Bennett, 1993)

$$
H_{0}^{(1)}(k R)=\frac{1}{\pi \mathrm{i}} \int_{-\infty}^{\infty} \frac{\mathrm{e}^{-\mathrm{i} \alpha(x-\xi)-\gamma|y-\eta|}}{\gamma} \mathrm{d} \alpha
$$

where $\gamma=\left(\alpha^{2}-k^{2}\right)^{\frac{1}{2}}=-\mathrm{i}\left(k^{2}-\alpha^{2}\right)^{\frac{1}{2}}$.

First of all let the singular point $P$ be at $(\xi, \eta), \eta>0$. Denote by $P^{\prime}$ the image point $(\xi,-\eta)$ and by $Q$ the field point $(x, y)$ and the distance between $P^{\prime}$ and $Q$ by

$$
R^{\prime}=\left[(x-\xi)^{2}+(y+\eta)^{2}\right]^{\frac{1}{2}}
$$

Write

$$
\begin{gathered}
\phi_{0}(x, y ; \xi, \eta)=H_{0}^{(1)}(k R)+\frac{1}{\pi \mathrm{i}} \int_{-\infty}^{\infty} A^{+}(\alpha) \mathrm{e}^{-\mathrm{i} \alpha x-\gamma y} \mathrm{~d} \alpha, \quad y>0, \\
\phi_{0}(x, y ; \xi, \eta)=\frac{1}{\pi \mathrm{i}} \int_{-\infty}^{\infty} A^{-}(\alpha) \mathrm{e}^{-\mathrm{i} \alpha x+\gamma y} \mathrm{~d} \alpha, \quad y<0
\end{gathered}
$$

each of which satisfy the Helmholtz equation by construction. Application of the barrier conditions (45) and (46) and use of the integral representation (50) yields

$$
A^{+}(\alpha)=\frac{\mathrm{e}^{\mathrm{i} \alpha \xi-\gamma \eta}}{2 \beta+\gamma}
$$

and

$$
A^{-}(\alpha)=\frac{2 \beta \mathrm{e}^{\mathrm{i} \alpha \xi-\gamma \eta}}{\gamma(2 \beta+\gamma)}
$$

and therefore

$$
\phi_{0}(x, y ; \xi, \eta)=H_{0}^{(1)}(k R)+\frac{\operatorname{sgn}(y)}{\pi \mathrm{i}} \int_{-\infty}^{\infty} \frac{\mathrm{e}^{-\mathrm{i} \alpha(x-\xi)-\gamma(|y|+\eta)}}{(2 \beta+\gamma)} \mathrm{d} \alpha, \quad \text { for all } y .
$$

Alternative representations are

$$
\phi_{0}(x, y ; \xi, \eta)=H_{0}^{(1)}(k R)+H_{0}^{(1)}\left(k R^{\prime}\right)-\frac{2 \beta}{\pi \mathrm{i}} \int_{-\infty}^{\infty} \frac{\mathrm{e}^{-\mathrm{i} \alpha(x-\xi)-\gamma(y+\eta)}}{\gamma(2 \beta+\gamma)} \mathrm{d} \alpha, \quad y>0,
$$


and

$$
\phi_{0}(x, y ; \xi, \eta)=\frac{2 \beta}{\pi \mathrm{i}} \int_{-\infty}^{\infty} \frac{\mathrm{e}^{-\mathrm{i} \alpha(x-\xi)+\gamma(y-\eta)}}{\gamma(2 \beta+\gamma)} \mathrm{d} \alpha, \quad y<0,
$$

and it is also noted that

$$
\frac{\partial \phi_{0}}{\partial y}(x, 0 ; \xi, \eta)=\frac{2 \beta}{\pi \mathrm{i}} \int_{-\infty}^{\infty} \frac{\mathrm{e}^{-\mathrm{i} \alpha(x-\xi)-\gamma \eta}}{2 \beta+\gamma} \mathrm{d} \alpha .
$$

A similar calculation with the source point at $(\xi,-\eta), \eta>0$, gives

$$
\phi_{0}(x, y ; \xi,-\eta)=H_{0}^{(1)}\left(k R^{\prime}\right)-\frac{\operatorname{sgn}(y)}{\pi \mathrm{i}} \int_{-\infty}^{\infty} \frac{\mathrm{e}^{-\mathrm{i} \alpha(x-\xi)-\gamma(|y|+\eta)}}{(2 \beta+\gamma)} \mathrm{d} \alpha, \quad \text { for all } y .
$$

It may readily be confirmed that

$$
\frac{\partial \phi_{0}}{\partial y}(x, 0 ; \xi,-\eta)=-\frac{\partial \phi_{0}}{\partial y}(x, 0 ; \xi, \eta)
$$

\section{Appendix II Numerical evaluation of the kernel}

When solving (36) for $W_{\alpha}\left(\hat{u}_{j}\right)$, it is useful to calculate the kernel by writing

$$
\frac{\partial \phi_{0}}{\partial y}(x, 0 ; \xi, 0)=\beta\left\{\phi_{0}\left(x, 0^{+} ; \xi, 0\right)-\phi_{0}\left(x, 0^{-} ; \xi, 0\right)\right\}=2 \beta\left\{H_{0}^{(1)}(k|x-\xi|)-K(\alpha)\right\},
$$

where equations (46), (57) and (58) have been used. Here

$$
\begin{aligned}
K(\alpha) & =\frac{2 \beta}{\pi \mathrm{i}} \int_{-\infty}^{\infty} \frac{\mathrm{e}^{-\mathrm{i} \alpha(x-\xi)}}{\gamma(2 \beta+\gamma)} \mathrm{d} \alpha=\frac{4 \beta}{\pi \mathrm{i}} \int_{0}^{\infty} \frac{\cos (\alpha X)}{\gamma(2 \beta+\gamma)} \mathrm{d} \alpha \\
& =\frac{4 \beta}{\pi \mathrm{i}}\left\{\int_{0}^{k} \frac{\mathrm{i} \cos (\alpha X)}{\mu(2 \beta-\mathrm{i} \mu)} \mathrm{d} \alpha+I(X)\right\} \\
& =\frac{4 \beta}{\pi \mathrm{i}}\left\{\int_{0}^{k} \frac{2 \beta \mathrm{i} \cos (\alpha X)}{\mu\left(4 \beta^{2}+\mu^{2}\right)} \mathrm{d} \alpha-\int_{0}^{k} \frac{\cos (\alpha X)}{\left(4 \beta^{2}+\mu^{2}\right)} \mathrm{d} \alpha+I(X)\right\}
\end{aligned}
$$

where $X=x-\xi, \gamma=\left(\alpha^{2}-k^{2}\right)^{\frac{1}{2}}=-\mathrm{i}\left(k^{2}-\alpha^{2}\right)^{\frac{1}{2}}=-\mathrm{i} \mu$, and

$$
I(X)=\int_{k}^{\infty} \frac{\cos (\alpha X)}{\gamma(2 \beta+\gamma)} \mathrm{d} \alpha=\int_{0}^{\infty} \frac{\cos (\alpha|X|)}{\alpha(2 \beta+\gamma)} \mathrm{d} \gamma=\operatorname{Re} \int_{0}^{\infty} \frac{\mathrm{e}^{\mathrm{i} \alpha|X|}}{\alpha(2 \beta+\gamma)} \mathrm{d} \gamma .
$$

An application of Cauchy's theorem in the first quadrant and some manipulation gives

$$
\begin{aligned}
I(X) & =\operatorname{Re} \int_{0}^{\mathrm{i} \infty} \frac{\mathrm{e}^{\mathrm{i} \alpha|X|}}{\alpha(2 \beta+\gamma)} \mathrm{d} \gamma=\operatorname{Re} \int_{0}^{\infty} \frac{\mathrm{ie}^{\mathrm{i} \alpha|X|}}{\alpha(2 \beta+\mathrm{i} \sigma)} \mathrm{d} \sigma \\
& =\operatorname{Re} \int_{0}^{k}\left(\frac{2 \mathrm{i} \beta+\sigma}{4 \beta^{2}+\sigma^{2}}\right) \frac{\mathrm{e}^{\mathrm{i} \alpha|X|}}{\rho} \mathrm{d} \sigma+\operatorname{Re} \int_{k}^{\infty}\left(\frac{2 \beta-\mathrm{i} \sigma}{4 \beta^{2}+\sigma^{2}}\right) \frac{\mathrm{e}^{\mathrm{i} \alpha|X|}}{\delta} \mathrm{d} \sigma \\
& =\int_{0}^{k} \frac{\sigma \cos (\rho X)}{\rho\left(4 \beta^{2}+\sigma^{2}\right)} \mathrm{d} \sigma-2 \beta \int_{0}^{k} \frac{\sin (\rho|X|)}{\rho\left(4 \beta^{2}+\sigma^{2}\right)} \mathrm{d} \sigma+2 \beta \int_{k}^{\infty} \frac{\mathrm{e}^{-\delta|X|}}{\delta\left(4 \beta^{2}+\sigma^{2}\right)} \mathrm{d} \sigma \\
& =\int_{0}^{k} \frac{\cos (\alpha X)}{\left(4 \beta^{2}+\mu^{2}\right)} \mathrm{d} \alpha-2 \beta \int_{0}^{k} \frac{\sin (\mu|X|)}{\mu\left(4 \beta^{2}+\alpha^{2}\right)} \mathrm{d} \alpha+2 \beta \int_{k}^{\infty} \frac{\mathrm{e}^{-\gamma|X|}}{\gamma\left(4 \beta^{2}+\alpha^{2}\right)} \mathrm{d} \alpha
\end{aligned}
$$


where $\rho=\left(k^{2}-\sigma^{2}\right)^{\frac{1}{2}}, \delta=\left(\sigma^{2}-k^{2}\right)^{\frac{1}{2}}$ and $\gamma=\mathrm{i} \sigma$ so that

$$
K(\alpha)=\frac{8 \beta^{2}}{\pi \mathrm{i}}\left\{\int_{0}^{k} \frac{\mathrm{i} \cos (\alpha X)}{\mu\left(4 \beta^{2}+\mu^{2}\right)} \mathrm{d} \alpha-\int_{0}^{k} \frac{\sin (\mu|X|)}{\mu\left(4 \beta^{2}+\alpha^{2}\right)} \mathrm{d} \alpha+\int_{k}^{\infty} \frac{\mathrm{e}^{-\gamma|X|}}{\gamma\left(4 \beta^{2}+\alpha^{2}\right)} \mathrm{d} \alpha\right\}
$$

which is straightforward to evaluate numerically. In each of the integrals in (66) the limit of integration $k$ may be changed to unity by scaling the integration variable.

\section{Appendix III References}

Carr, J. H. and Stelzriede, M. E. (1952) "Diffraction of water waves by breakwaters." US Nat. Bur. Stds., 521, 109-125.

Gilbert, G. and Brampton, A. H. (1985) "The solution of two wave diffraction problems using integral equations." Technical Report IT 299, Hydraulics Research, Wallingford.

Hunt, B. (1990) "An integral equation solution for a breakwater gap problem." J. Hydraulic. Research, 28, 609-619.

Jones, D. S. (1986) "Acoustic and Electromagnetic Waves.” Oxford University Press.

Lynett, P. J., Liu, P. L.-F., Losado, I. J. and Vidal, C. (2000) "Solitary wave interaction with porous breakwaters." J. Wtrwy., Port, Coast. and Ocean Engrg, ASCE, 126, 314-322.

McIver, P. (1999) "Water-wave diffraction by thin porous breakwaters." J. Wtrwy., Port, Coast. and Ocean Engrg, ASCE, 125, 66-70.

McIver, P. and Bennett, G. S. (1993) "Scattering of water waves by axisymmetric bodies in a channel." J. Eng. Math., 27, 1-29.

Noble, B. (1988) "Methods Based on the Wiener-Hopf Technique." 2nd edition, Chelsea Publishing Company, New York.

Penney, W. G. and Price, A. T. (1952) "The diffraction theory of sea waves and the shelter afforded by breakwaters." Phil. Trans. Royal Soc. Lond. A, 244, 236-253.

Porter, D. and Chu, K.-W. E. (1986) "The solution of two wave-diffraction problems". J. Eng. Math., 20, 63-72. 
Sollitt, C. K. and Cross, R. H. (1972) "Wave transmission through porous breakwaters." Proc., 13th Conf. on Coast. Engrg., ASCE, 1827-1846.

Yu, X. (1995) "Diffraction of water waves by porous breakwaters." J. Wtrwy., Port, Coast. and Ocean Engrg, ASCE, 121, 275-282.

Yu, X. and Togashi, H. (1996) "Combined diffraction and transmission of water waves around a porous breakwater gap." Proc., 25th Conf. on Coast. Engrg., ASCE, 2063-2076. 

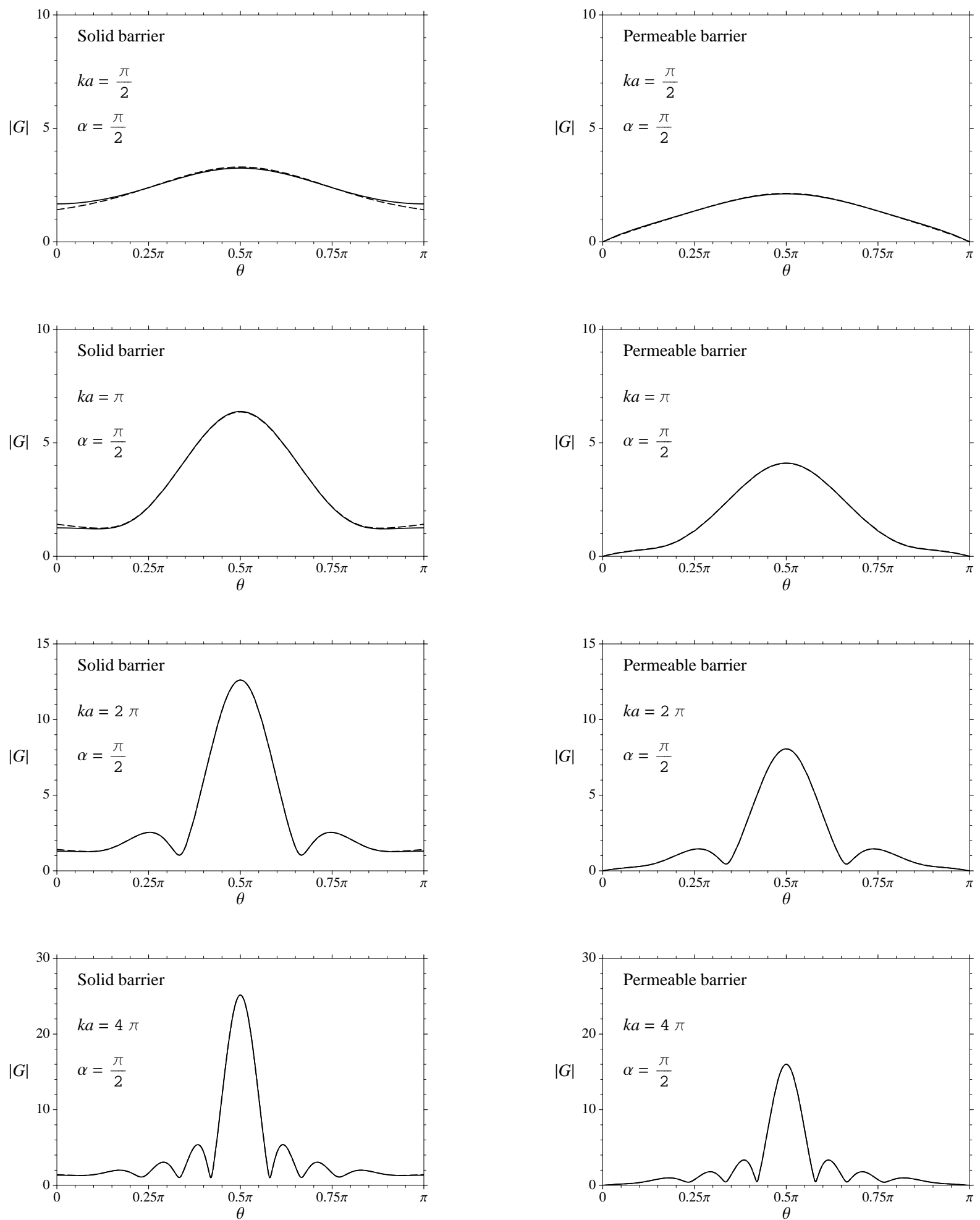

Figure 2: Modulus of the diffraction coefficient $|G(\theta, \alpha)|$ vs. $\theta$ for $\alpha=\pi / 2$ : full solution ( $\square$, large-gap approximation $(----)$. 

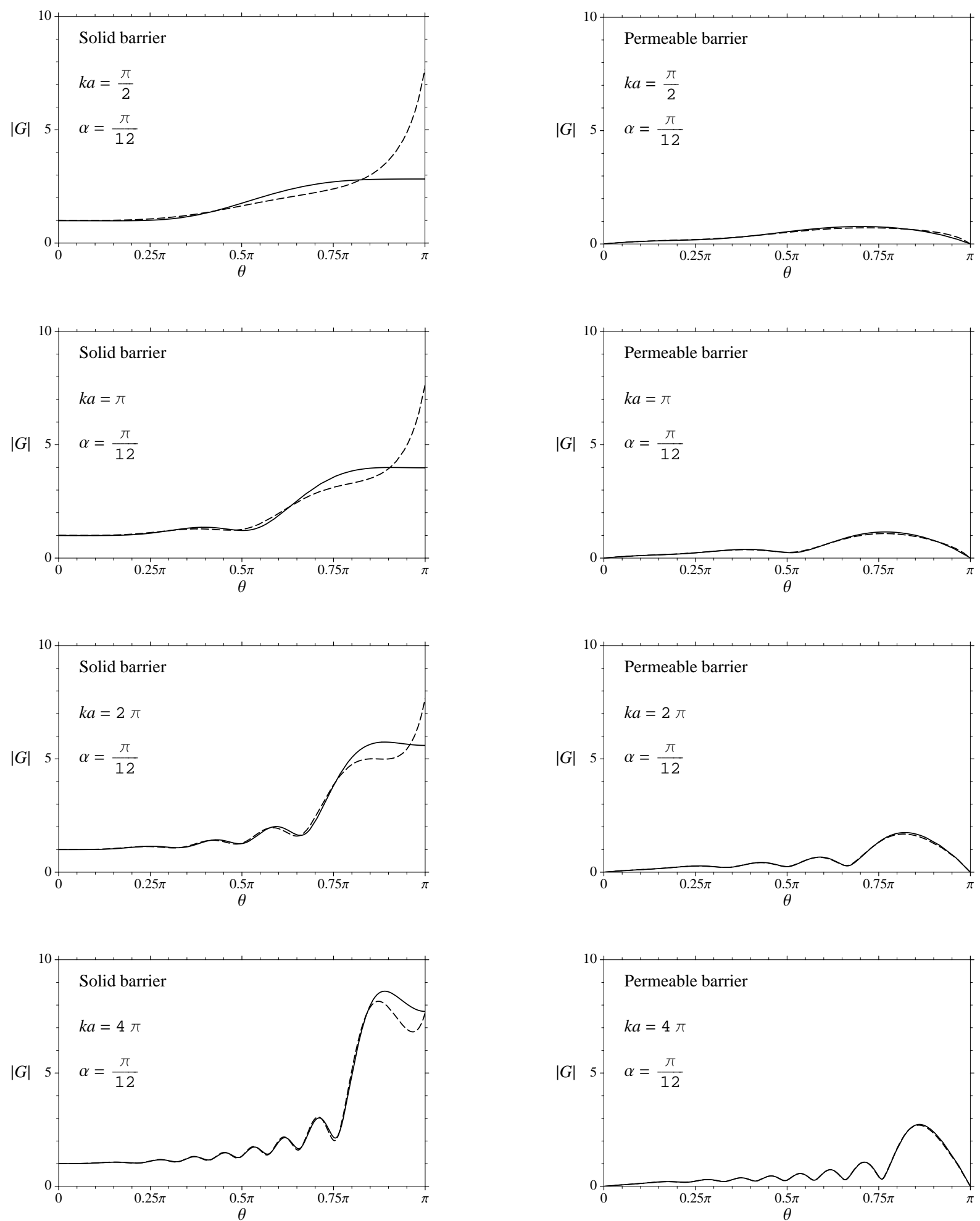

Figure 3: Modulus of the diffraction coefficient $|G(\theta, \alpha)|$ vs. $\theta$ for $\alpha=\pi / 12$ : full solution ( $\square$, large-gap approximation (--- -). 